\title{
Multimodality imaging of coronavirus disease 2019 (COVID-19): a pictorial essay
}

\author{
Babalola I. Afolabi iB,D,E,F, Bukunmi M. Idowu ${ }^{2 A, B, D, E, F}$, Stephen 0. Onigbinde ${ }^{3 B, D, E, F}$ \\ 'Department of Radiology, Obafemi Awolowo University Teaching Hospitals Complex, lle-Ife, Osun State, Nigeria \\ 2Department of Radiology, Union Diagnostics and Clinical Services PIc, Yaba, Lagos State, Nigeria \\ ${ }^{3}$ Department of Anatomical sciences, School of Medicine, St. George's University, Grenada
}

\section{Abstract}

The coronavirus disease 19 (COVID-19) or severe acute respiratory syndrome coronavirus 2 (SARS-CoV-2) or new coronavirus 2019 (2019-nCoV) is now a global pandemic with attendant morbidity, mortality, and socioeconomic disruption. Its features have been described on plain chest radiography, chest computed tomography (CT), chest ultrasonography, brain CT, brain magnetic resonance imaging, and fluorodeoxyglucose-positron emission tomography. Based on current evidence, imaging plays an ancillary role in the management of those with severe illness. This essay illustrates the imaging manifestations of COVID-19 pictorially.

Key words: severe acute respiratory syndrome coronavirus 2 (SARS-CoV-2), COVID-19, computerised tomography, chest radiography, ultrasonography, imaging.

\section{Introduction}

There has been a massive outbreak of a novel coronavirus infection since December 2019 [1]. The virus, presently known as severe acute respiratory syndrome coronavirus 2 (SARS-CoV-2) or coronavirus disease 19 (COVID-19) or new coronavirus 2019 (2019-nCoV), manifests with respiratory disease of variable severity. It attained pandemic proportions according to the World Health Organisation on March 11, 2020 [1].

The typical symptoms of COVID-19 infection are fever, dry cough, fatigue, sputum production, shortness of breath, myalgia or arthralgia, sore throat, headache, chills, nausea or vomiting, nasal congestion, diarrhoea, haemoptysis, and conjunctival congestion [1]. COVID-19 infection is confirmed using the reverse transcriptase polymerase chain reaction (RT-PCR) test [1].

Currently, imaging is not recommended for screening or diagnosis of COVID-19 infection [2-4]. Imaging should not be used to determine the need to test a pa- tient for COVID-19. A normal chest radiograph or chest computed tomography (CT) does not exclude COVID-19 infection, while an abnormal study is not pathognomonic for COVID-19 diagnosis [2-4]. Imaging should be used to only answer definite clinical questions in symptomatic in-patients (e.g. suspected pulmonary embolism, distinguishing organising pneumonia from acute respiratory distress syndrome [ARDS], excluding stroke or encephalopathy in those with neurological symptoms, etc.) [2-4].

Radiologists and other physicians need to be well acquainted with the radiological features of COVID-19 infection because the disease may be encountered in patients imaged for other indications. The purpose of this review is to illustrate the imaging manifestations of COVID-19 pictorially.

\section{Chest radiography}

Chest radiography is readily available and affordable. Though it has limited sensitivity for COVID-19 infection,

\footnotetext{
Correspondence address:

Dr. Bukunmi M. Idowu, Department of Radiology, Union Diagnostics and Clinical Services Plc, 37 Tejuosho Rd, Yaba, Lagos State, Nigeria,

e-mail: ibmcontacts@gmail.com
}

Authors' contribution:

A Study design · B Data collection · C Statistical analysis · D Data interpretation - E Manuscript preparation · F Literature search · G Funds collection 
it is still a first-line modality that can also be used for follow-up and evaluation of complications [2]. The common findings on chest radiography (consolidation, ground-glass opacities) are usually bilateral and peripheral, with lower zone predominance [5]. There have also been reports of spontaneous pneumomediastinum. Unfortunately, chest radiographs may be normal in the early stages or mild forms of the disease. Figures 1-6 illustrate the plain chest radiographic features of RT-PCR-confirmed COVID-19 infection.

\section{Chest computerised tomography}

Non-contrast (plain) chest CT at 0.625-1.5 mm slice thickness is the standard protocol for COVID-19 pa-

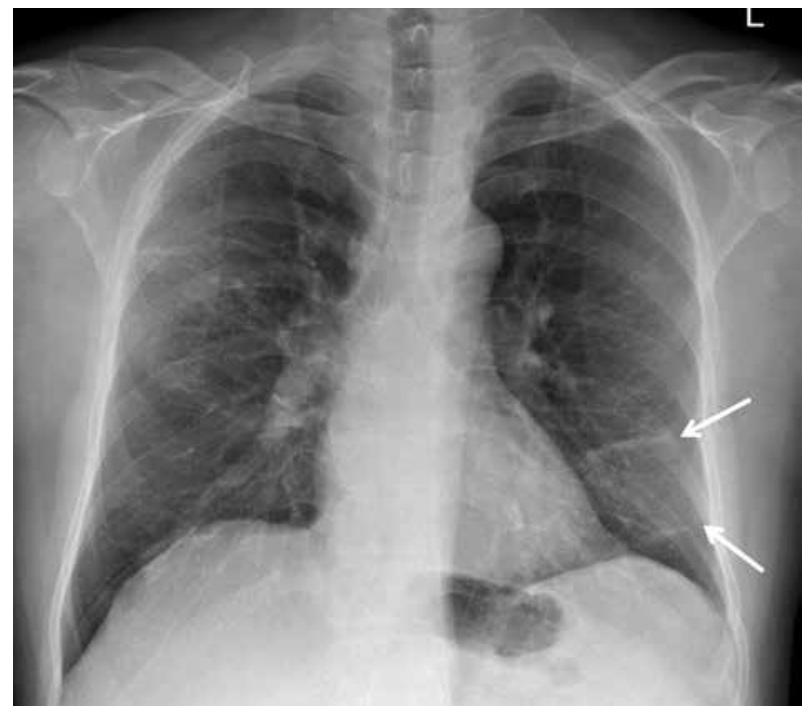

Figure 1. Posteroanterior chest radiograph of a 56-year-old COVID-19positive man, showing streaky opacities in the left lower lung zone (arrows)

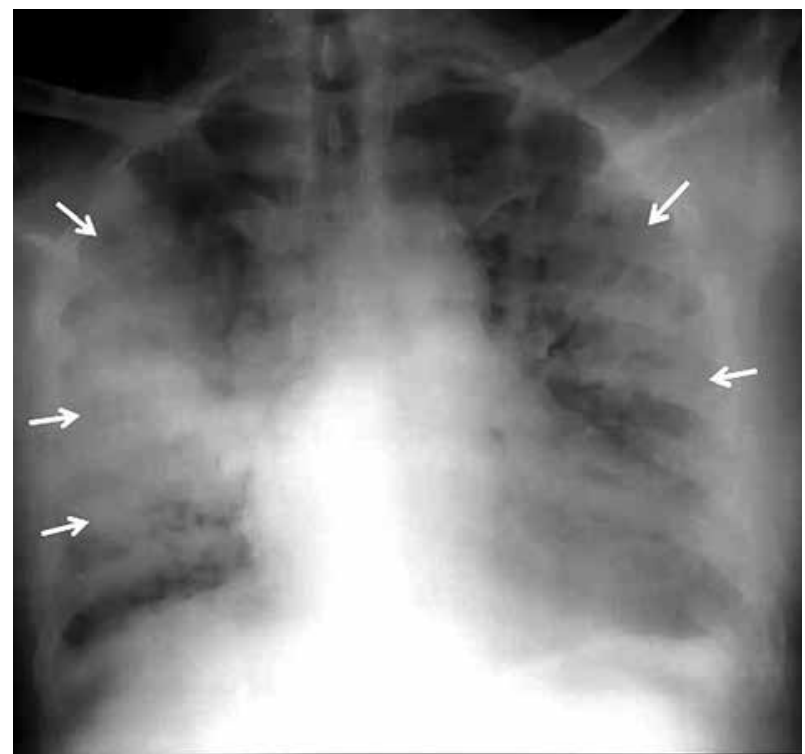

Figure 3. Anteroposterior chest radiograph of a 65 -year-old COVID-19positive man, showing extensive ground glass opacities and multifocal areas of consolidations (arrows) in bilateral (right > left) middle and lower zones tients. A pre-contrast study is necessary for those who require contrast administration (e.g. suspected pulmonary embolism, history of haemoptysis); otherwise, accurate interpretation of ground-glass opacities might be difficult [6].

The notable findings on chest CT are depicted below.

\section{Ground-glass opacities}

Ground glass opacities (GGO) are foggy opacities that do not conceal the underlying bronchial structures or pulmonary vessels (Figures 7 and 8). GGO maybe as a result of partial exudate or transudate filling the air spaces of the lungs, as well as an interstitial thickening or partial collapse of the alveoli [7]. In COVID-19 patients, GGO

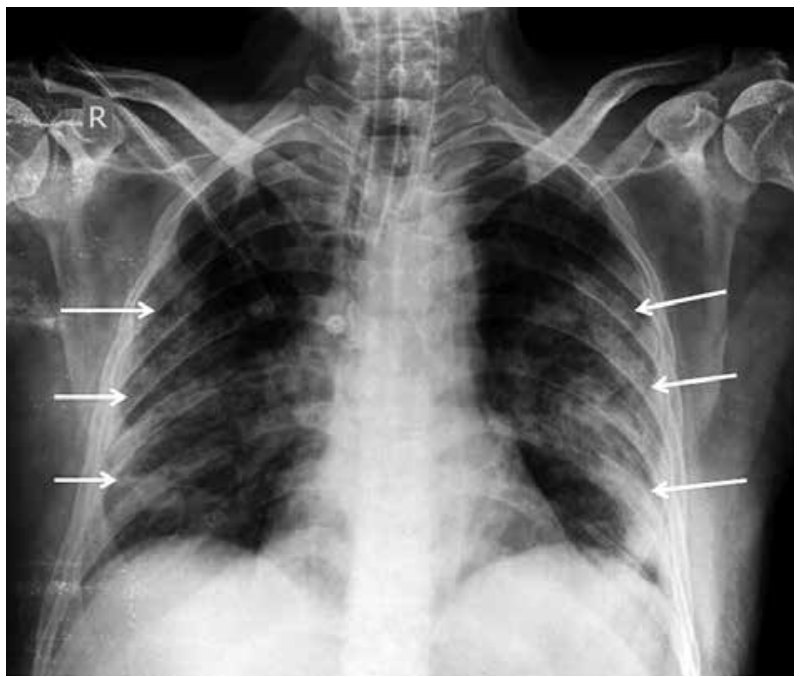

Figure 2. Posteroanterior chest radiograph of a 45-year-old COVID-19-positive man, showing bilateral ground-glass opacities more peripherally and predominantly in the mid and lower lung zones. An endotracheal tube is in-situ

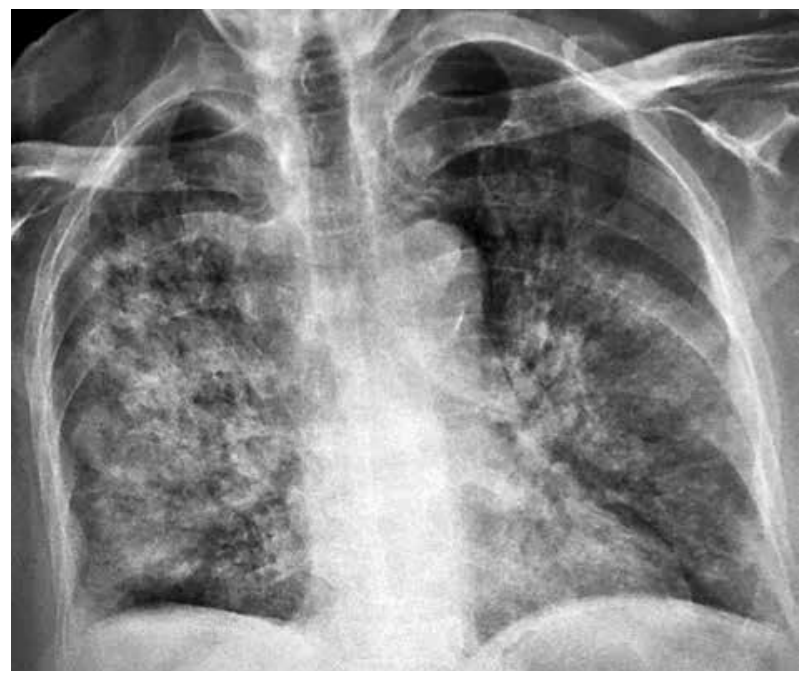

Figure 4. Chest radiograph (AP view) of a 49-year-old COVID-19-positive man. There are diffuse bilateral consolidations (more on the right) with scattered areas of ground glass opacities (more in the left lung). There is associated air bronchogram within the opacities. Relative sparring of both lung apices is noted. No pleural effusion 


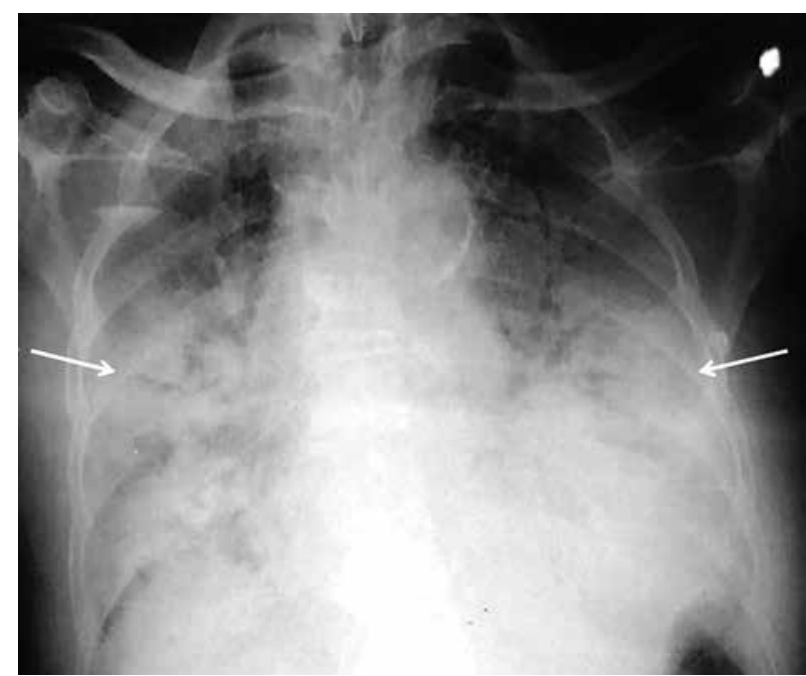

Figure 5. Chest radiograph (AP view) in a 59-year-old COVID-19-positive man, showing extensive bilateral pulmonary consolidation with air bronchograms

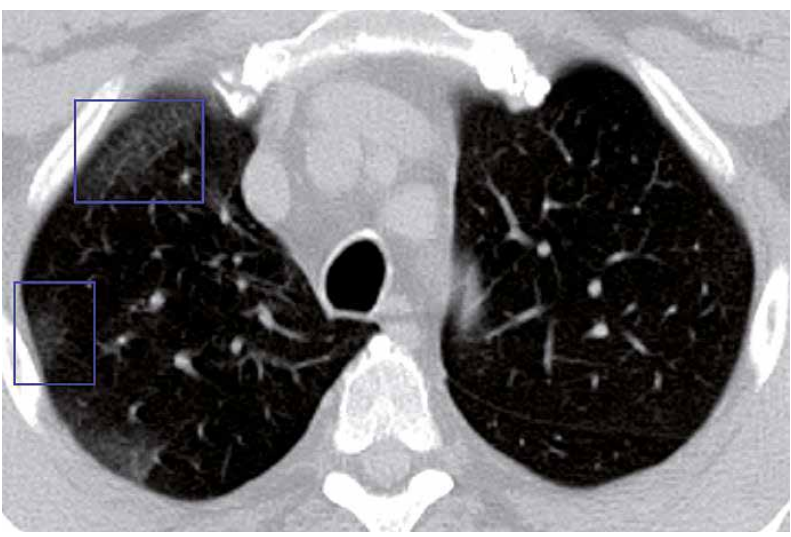

Figure 7. Non-contrast axial chest computed tomography image of a 65 -year-old COVID-19-positive male with cough and fever, showing pure ground-glass opacities in the subpleural areas (zoom boxes)

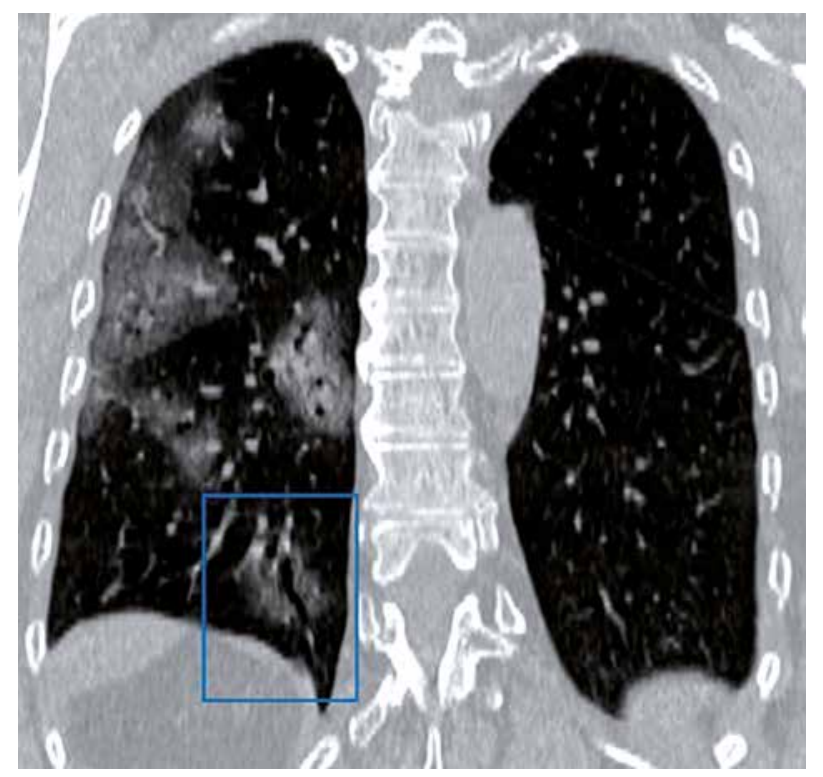

Figure 8. Non-contrast chest computed tomography coronal reformat of a 67-year-old COVID-19-positive male with cough, fever, and shortness of breath showing multiple right-sided ground glass opacities and air bronchogram in the right lower lobe (zoom box)
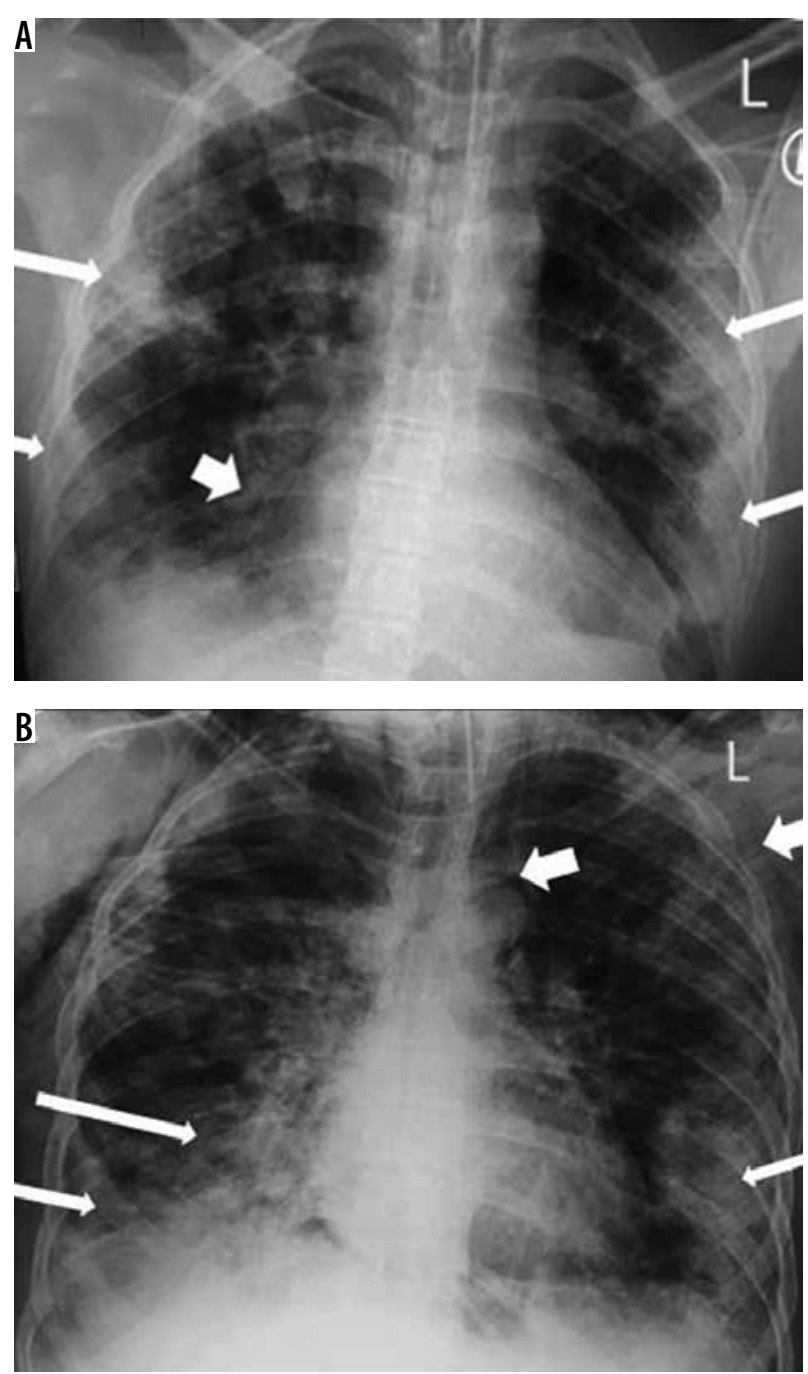

Figure 6. The above pair of (XR (AP view) is for a 38-year-old man with COVID-19 infection, who was examined twice, four days apart. On day 1 (A), there are predominantly peripheral opacities bilaterally (thin arrows) and right paracardiac consolidation with an air-bronchogram (thick arrow). Follow-up radiograph on day 5 (B) shows a progression of the ground-glass opacities and consolidations (thin arrows) with development of spontaneous pneumomediastinum and subcutaneous emphysema (thick arrows)

is usually bilateral, multilobar, peripherally or posteriorly distributed (or both), and usually in the lower lobes.

\section{Consolidation}

Consolidation occurs when pathological fluids, tissues, or cells replace alveolar air, causing opacification due to the increase of pulmonary parenchymal density obscuring the underlying vessels (Figure 9) [7,8] Consolidation often becomes the second most predominant finding about 14 days after the onset of the disease [7]. It could be patchy or multifocal, and its presence may indicate increased severity [8].

\section{Air bronchogram}

Air space consolidations are air- or gas-filled bronchi surrounded by grey or white alveoli made visible on a back- 


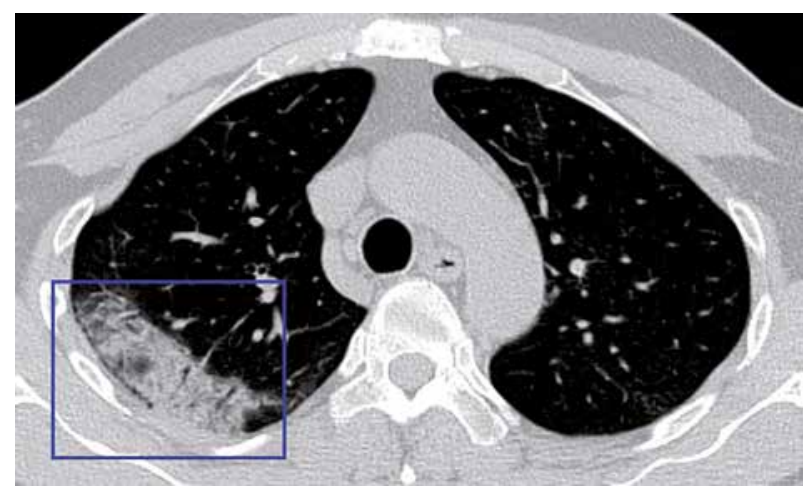

Figure 9. Non-contrast axial chest computed tomography image of a 56-yearold COVID-19-positive male with cough and shortness of breath, showing consolidation with air bronchogram in the subpleural area (zoom box)

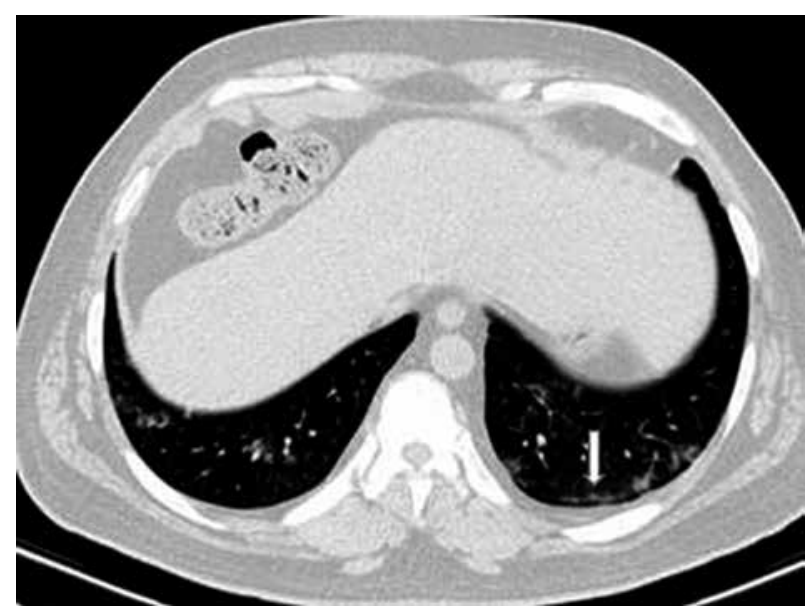

Figure 11. Non-contrast axial chest computed tomography image of a 42-year-old COVID-19-positive male with cough and shortness of breath, depicting a subpleural line (zoom box)

ground of opaque (high attenuation) airless lung (Figure 9) $[7,8]$. Air bronchogram characterises air space consolidation.

\section{Nodules}

Nodules are rounded irregular, poorly defined opacities that measure about $3 \mathrm{~cm}$ diameter [8]. They can be micronodular or macronodular. They can also be classified into discrete focal nodules and centrilobular or 'tree in bud nodules'. Nodules have been reported in 3-13\% of COVID-19 patients, appearing as multifocal solid irregular nodules (Figure 10), sometimes with a visible halo sign $[4,9]$. However, centrilobular nodules are infrequent in COVID-19.

\section{Pleura affectation}

Pleural thickening (common; seen in about 56\%) and pleural effusion (uncommon) may be present in COVID-19 $[3,9]$. The presence of pleural effusion should alert to the possibility of underlying immunocompromised status [10].

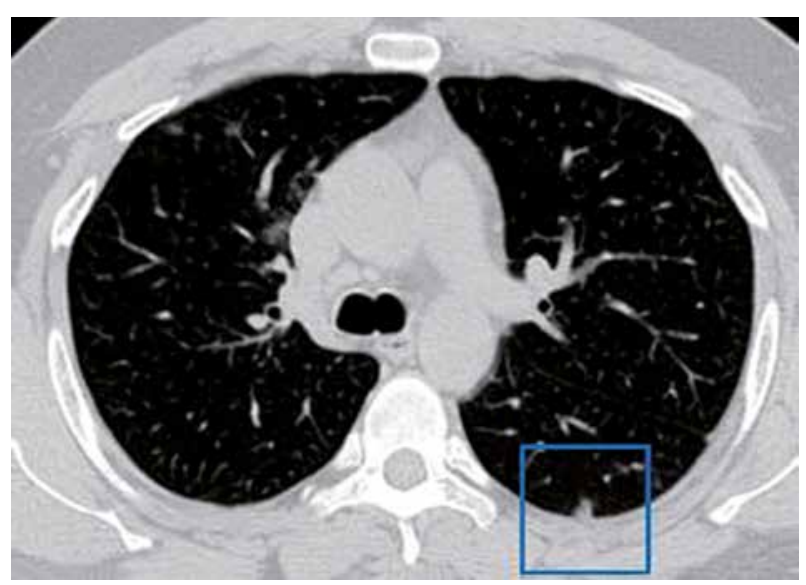

Figure 10. Non-contrast axial chest computed tomography image of a 38-year-old COVID-19-positive male with cough and fever, showing a subpleural nodule (zoom box)

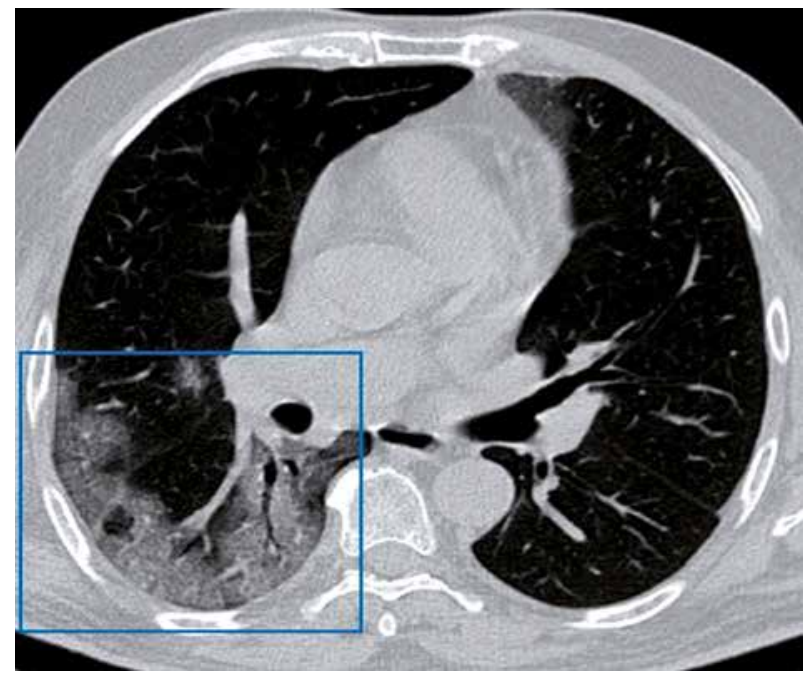

Figure 12. Non-contrast axial chest computed tomography image of a 67-year-old COVID-19-positive male with fever, cough, and shortness of breath, showing a reticular pattern in the subpleural areas of groundglass opacities (zoom box)

\section{Subpleural lines}

The subpleural line is a thin curvilinear opacity of about 1-3 $\mathrm{mm}$ in thickness found close to the pleural surface (Figures 11 and 12) [11]. It is located in the subpleural region and is distributed parallel to the pleural surface. It is a non-specific sign, seen in $33.9 \%$ of COVID-19 cases, but can also be caused by dependent atelectasis, pulmonary oedema, and fibrosis [11]. Subpleural lines may be related to the predominantly peripheral location of parenchymal changes in COVID-19.

\section{Fibrosis}

Fibrotic lesions may occur due to the replacement of cellular components by scar tissues in patients with proliferative diseases and patients recovering from chronic pulmonary inflammation (Figure 13) [9]. Fibrosis in COVID-19 is usually not seen at the early stage until 

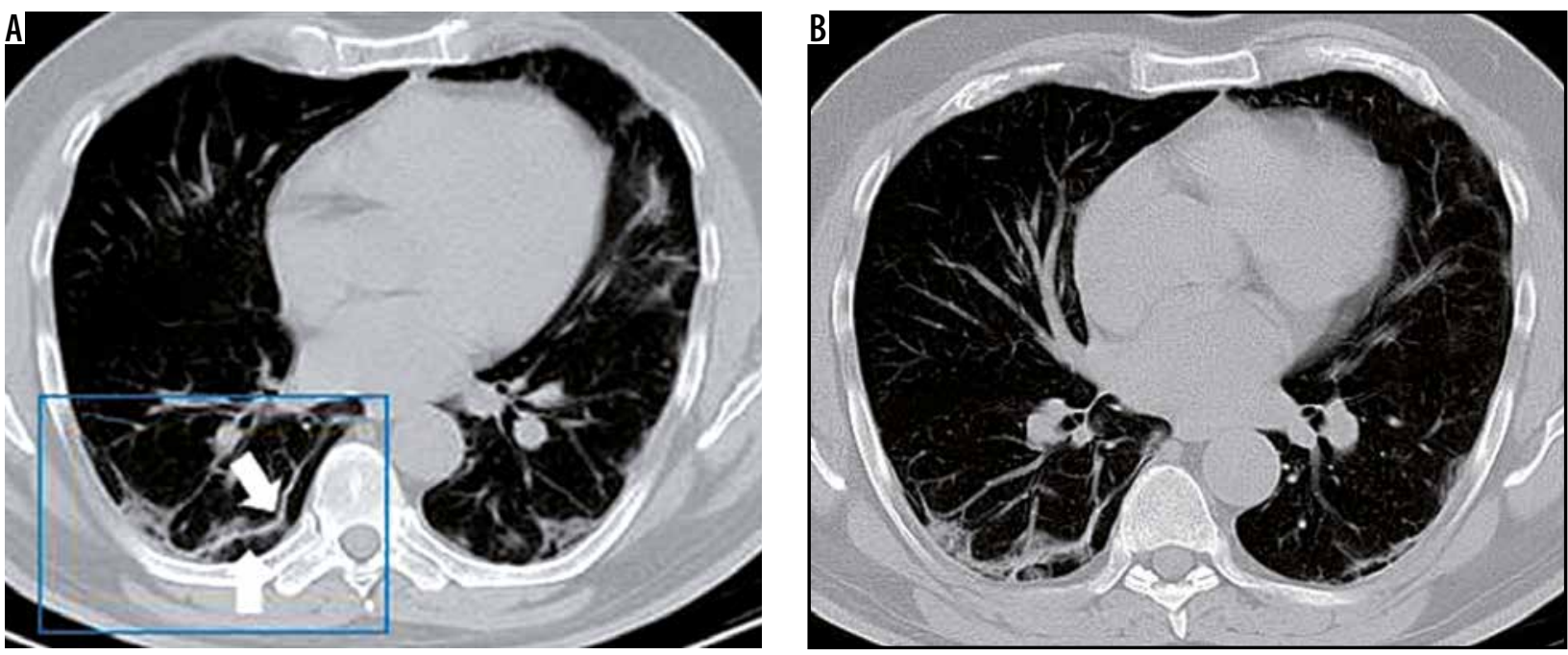

Figure 13. Non-contrast axial chest computed tomography image of a 60-year-old COVID-19-positive male with cough, depicting subpleural ground-glass opacities with fibrous stripes (arrows within zoom box)

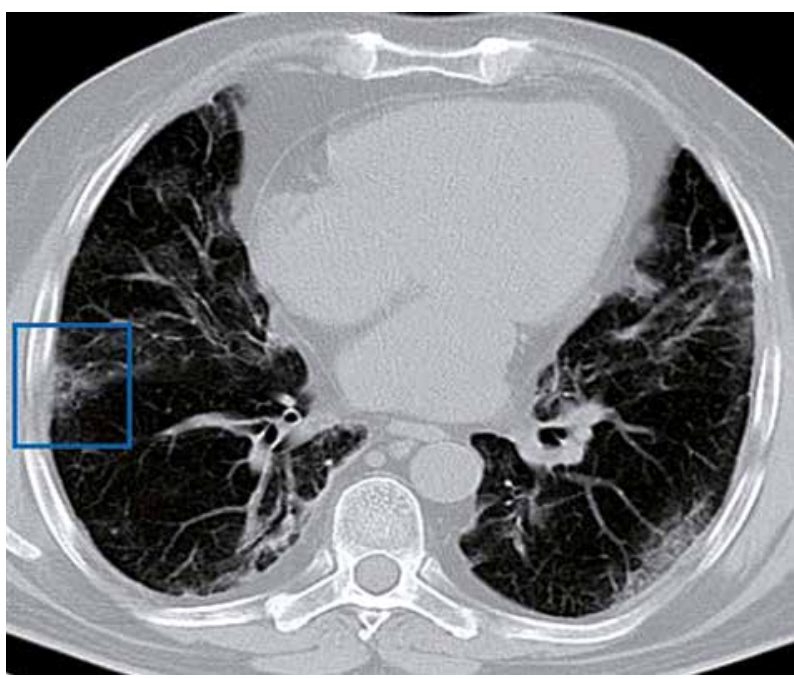

Figure 14. Non-contrast axial chest computed tomography image of a 70-year-old COVID-19-positive male with fever, cough, and shortness of breath, showing reversed halo (atoll) sign (zoom box)

2-3 weeks when interstitial changes begin to occur [4] Some studies argued that the presence of fibrosis indicates advanced COVID-19 [12], which could lead to bronchus deformation. In contrast, others opined that it depicts healing [9].

\section{Halo sign and atoll sign}

The halo sign comprises masses that surround GGO [12]. It is related to perilesional haemorrhage in angioinvasive fungal infections, hypervascular metastases, viral infections, and organising pneumonia [12]. However, the pathogenesis of halo sign in COVID-19 is unknown [12]. Halo sign, together with other characteristic manifestations (vascular thickening, air bronchogram sign, and crazy-paving pattern), is usually associated with GGO at the early onset of COVID-19 [12]. Reversed halo sign may indicate a developed stage of COVID-19, and it is associated

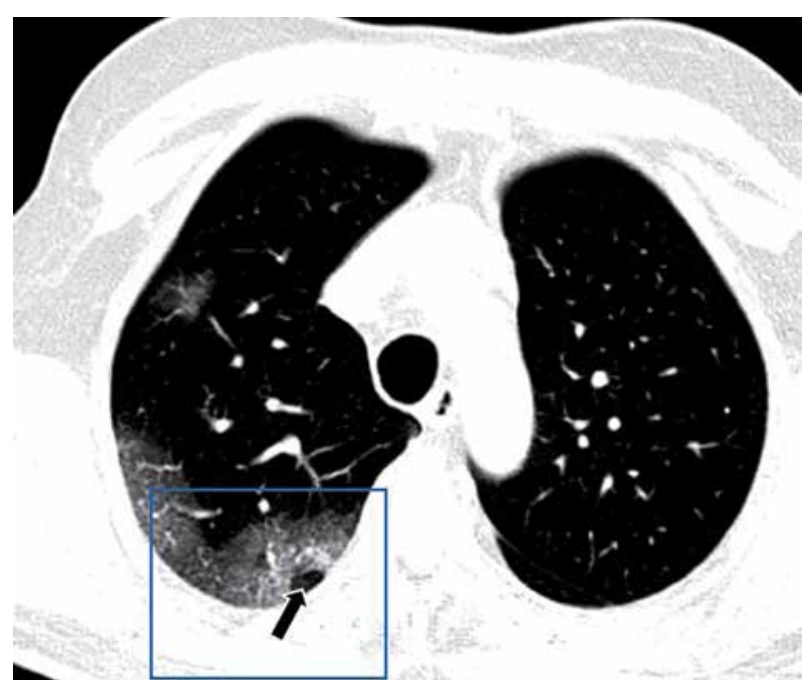

Figure 15. Non-contrast axial chest computed tomography image of a 67-yearold COVID-19-positive male with fever, cough, and shortness of breath, showing ground-glass opacity with air bubble sign (arrow and zoom box)

with the consolidation of GGO [12]. On CT, a "reversed halo sign (atoll sign)" (Figure 14) and/or pulmonary nodules with a halo sign may be seen in COVID-19 [9].

\section{Cavitation and air bubble sign}

Cavitation is notably uncommon in COVID-19 patients [7]. However, air bubble sign or bubble-like air-containing space, which is a small air-containing space associated with bronchiolectasis (Figure 15), has been termed "cavities" by some authors [12].

\section{Airway}

Bronchiectasis and bronchial wall thickening are features of COVID-19 infection (Figure 16) [12]. Bronchial wall thickening is predominant in more critical COVID-19 cases compared to mild cases [12]. They are caused by 


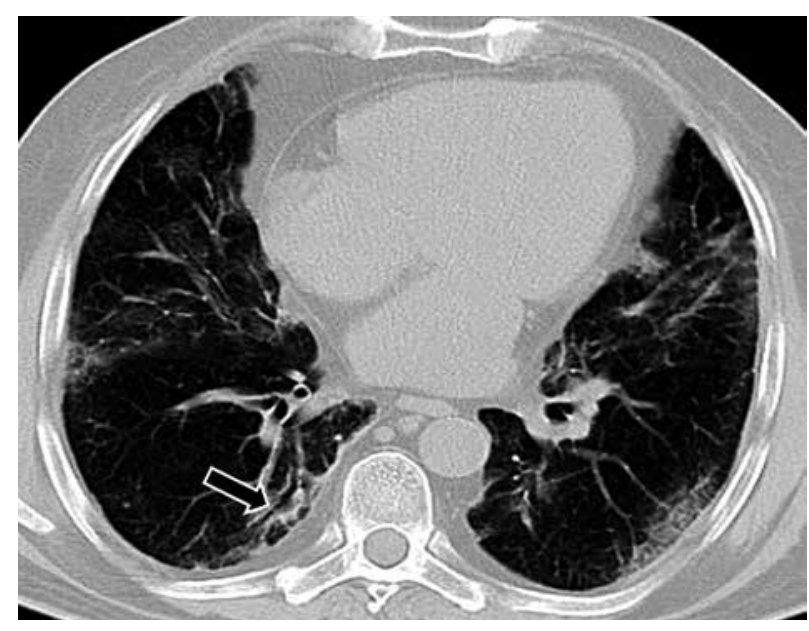

Figure 16. Non-contrast axial chest computed tomography image of a 70-year-old COVID-19-positive male with fever, cough, and shortness of breath, showing bilateral lower lobe reticular pattern, ground-glass opacity, and bronchial wall thickening (black arrow)

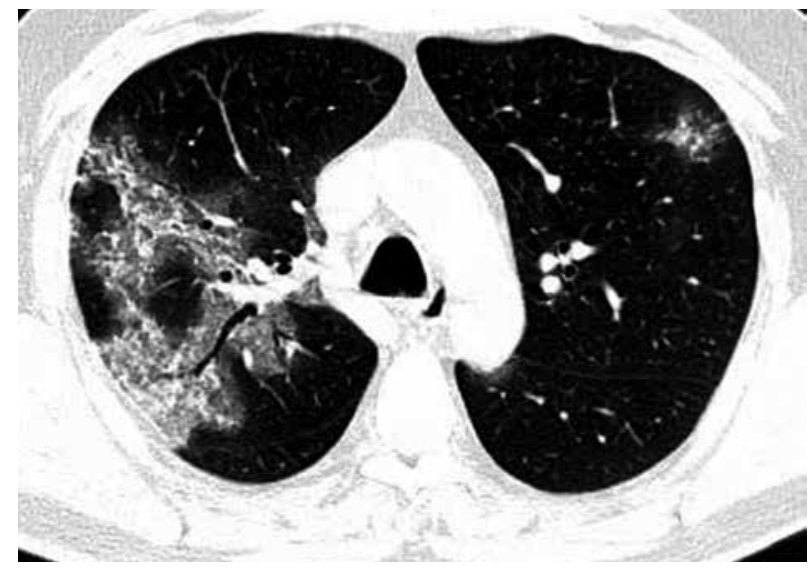

Figure 17. Non-contrast axial chest computed tomography image of a 64-year-old COVID-19-positive male with cough and shortness of breath, showing reticular pattern superimposed on the background of ground-glass opacity, giving the crazy-paving pattern

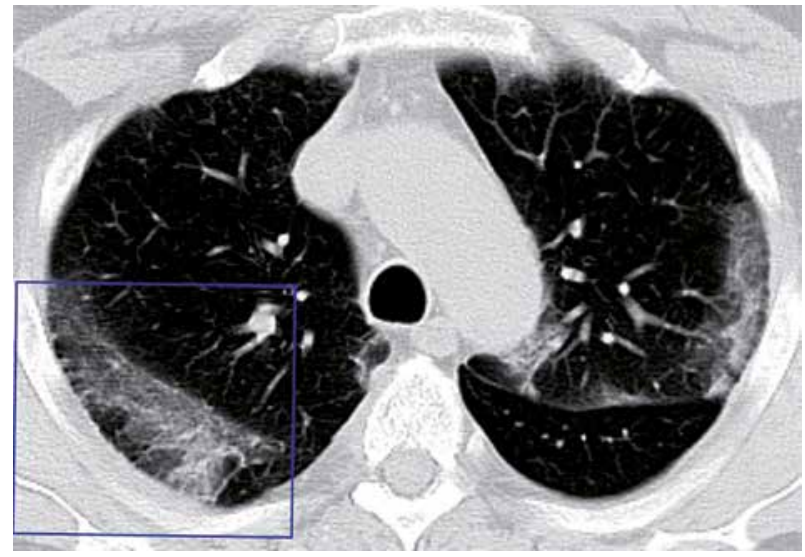

Figure 18. Non-contrast axial chest computed tomography image of a 60-year-old COVID-19-positive male with cough, showing reticular pattern superimposed on background ground-glass opacity

bronchial obstruction and inflammatory damage of the bronchial wall, which leads to tractive bronchiectasis, fibrosis, fibrous tissue proliferation, and destruction of the bronchial wall structure [12].

\section{Crazy-paving appearance}

The crazy-paving pattern is produced by ground-glass opacity overlaid on a web of linear opacities. The linear opacification may be due to superimposed thickened intralobular lines and interlobular septa (Figures 17 and 18) [9]. It is rare in COVID-19, where it could characterise a developed stage at about the third week after the onset of symptoms [9].

\section{Bronchiectasis}

Bronchiectasis and bronchial wall thickening are airway changes, which may be due to obstruction of the bronchi (damaging the structure of the wall), inflammation of the bronchial wall, proliferation of fibrous tissue, tractive bronchiectasis, and fibrosis. Bronchiectasis is seen in $10-20 \%$ of COVID-19 patients [8].

\section{Vascular changes}

Vascular dilatation or widening of the pulmonary vessels is a typical feature within GGO [12]. It may be caused by pro-inflammatory factors, which result in damage and oedema of the capillary wall [12]. It is observed as early as the first week of onset but does not exhibit the irregular dilatation and convergence seen in malignant lesions [12].

\section{Lymphadenopathy}

Lymphadenopathy may manifest as early as the first week in some COVID-19 patients, and towards the third week in others [9]. Its occurrence alongside tiny nodules and pleural effusion may signify a bacterial superinfection. Lymphadenopathy has been reported in $4-8 \%$ of patients. It is a marker of severity [9].

\section{Imaging differential diagnoses of COVID-19 infection}

The common differentials include viral pneumoniae, atypical pneumonia (Chlamydophila pneumoniae, Mycoplasma pneumoniae, Legionella pneumophilia), acute eosinophilic pneumonia, acute interstitial pneumonia, cryptogenic organising pneumonia, acute pulmonary oedema (cardiogenic and non-cardiogenic), and interstitial lung disease [6].

Ground-glass opacity ( $91 \%$ vs. $68 \%$ ), peripheral distribution ( $80 \%$ vs. $57 \%$ ), vascular thickening $(59 \%$ vs. $22 \%$ ), and fine reticular opacity ( $56 \%$ vs. $22 \%$ ) are more predominant in COVID-19 pneumonia than in nonCOVID-19 pneumoniae, whereas pleural effusion $(4.1 \%$ vs. $39 \%)$, lymphadenopathy ( $2.7 \%$ vs. $10.2 \%)$, and concur- 
rent central and peripheral distribution of lesions (14\% vs. $35 \%$ ) are less common in COVID-19 pneumonia than in non-COVID-19 pneumoniae [13].

\section{Chest ultrasonography}

The stage of the disease, extent of lung damage, and the presence of comorbidities determine the sonographic features of COVID-19 pneumonia. The posterobasal aspects of the lung fields are the most common sites of COVID-19 affectation on ultrasonography $[6,14]$.

The sonographic features of COVID-19 pneumonia include irregularly thickened and discontinuous pleural line, the appearance of B lines (focal, multifocal, or confluent), subpleural consolidations, alveolar consolidation with static or mobile sonographic air bronchograms/air bronchiolograms, and return of bilateral A lines during recuperation (restoration of aeration during recovery) $[6,14]$.

\section{Neuroimaging in COVID-19}

A neurological complication of COVID-19 infection that may require brain imaging is acute haemorrhagic necrotising encephalopathy (AHNE) [15]. AHNE lesions are hypodense on CT and hyperintense on T2 FLAIR MRI with internal haemorrhage. Ring enhancement may be seen after intravenous contrast [15]. The lesions are characteristically symmetric, multifocal, and always involve the thalamus. The supratentorial white matter, cerebellum, and brain stem may also be affected [15]. Cerebrovascular accident (stroke) has also been reported in patients with COVID-19 infection [16].

\section{PET-CT hybrid imaging}

The pulmonary ground-glass opacities in COVID-19 show increased avidity for 18-fluorodeoxyglucose (FDG). Furthermore, patients whose pulmonary lesions exhibit higher standardised uptake value (SUV) may take longer to recover [6].

\section{Conclusion}

Imaging plays a supportive role in the care of COVID-19 patients. The imaging manifestations are diverse and depend on the stage of the disease. Chest CT is the most informative imaging modality for assessing its pulmonary manifestations.

\section{Conflict of interest}

The authors report no conflict of interest.

\section{References}

1. Jin H, Hong C, Chen S, et al. Consensus for prevention and management of coronavirus disease 2019 (COVID-19) for neurologists. Stroke Vasc Neurol 2020; 5: 146-151.

2. Yoon SH, Lee KH, Kim JY, et al. Chest radiographic and CT findings of the 2019 novel coronavirus disease (COVID-19): analysis of nine patients treated in Korea. Korean J Radiol 2020; 21: 494-500.

3. $\mathrm{Xu} \mathrm{X}, \mathrm{Yu} \mathrm{C}, \mathrm{Qu}$ J, et al. Imaging and clinical features of patients with 2019 novel coronavirus SARS-CoV-2. Eur J Nucl Med Mol Imaging 2020; 47: 1275-1280

4. Shi H, Han X, Jiang N, et al. Radiological findings from 81 patients with COVID-19 pneumonia in Wuhan, China: a descriptive study. Lancet Infect Dis 2020; 20: 425-434.

5. Rodrigues JCL, Hare SS, Edey A, et al. An update on COVID-19 for the radiologist - a British Society of Thoracic Imaging statement. Clin Radiol 2020; 75: 323-325.

6. Knipe H, Bell DJ. COVID-19 | Radiology Reference Article | Radiopaedia.org [Internet]. Radiopaedia. Available at: https://radiopaedia. org/articles/covid-19-3?lang=gb (Accessed: 4.04.2020).

7. Ye Z, Zhang Y, Wang Y, Huang Z, Song B. Chest CT manifestations of new coronavirus disease 2019 (COVID-19): a pictorial review. Eur Radiol 2020; 30: 4381-4389.

8. Agarwal PP, Cinti S, Kazerooni EA. Chest radiographic and CT findings in novel swine-origin influenza A (H1N1) virus (S-OIV) infection. AJR Am J Roentgenol 2009; 193: 1488-1493.

9. Li Y, Xia L. Coronavirus disease 2019 (COVID-19): role of chest CT in diagnosis and management. AJR Am J Roentgenol 2020; 214: $1280-1286$.
10. Pan F, Ye T, Sun P, et al. Time course of lung changes on chest CT during recovery from 2019 novel coronavirus (COVID-19) Pneumonia. Radiology 2020; 200370. DOI: 10.1148/radiol.2020200370.

11. Silva CIS, Marchiori E, Souza Júnior AS, Müller NL. Illustrated Brazilian consensus of terms and fundamental patterns in chest CT scans. J Bras Pneumol 2010; 36: 99-123.

12. Wang Y, Dong C, Hu Y, et al. Temporal changes of CT findings in 90 patients with COVID-19 pneumonia: a longitudinal study. Radiology 2020; 200843. DOI: 10.1148/radiol.2020200843.

13. Amanullah S, Shapiro ML, Dinescu DV, Lin EC, Singh SP. Viral pneumonia imaging: practice essentials, radiography, computed tomography [Internet]. 2020 Available from: https://emedicine.medscape.com/article/360254-overview\#showall (Accessed: 26.04.2020).

14. Peng QY, Wang XT, Zhang LN. Findings of lung ultrasonography of novel corona virus pneumonia during the 2019-2020 epidemic. Intensive Care Med 2020; DOI: 10.1007/s00134-020-05996-6.

15. Poyiadji N, Shahin G, Noujaim D, Stone M, Patel S, Griffith B. COVID-19-associated acute hemorrhagic necrotizing encephalopathy: CT and MRI features. Radiology 2020; 201187. DOI: 10.1148/radiol. 2020201187.

16. Atakla AG, Conde K, Neishay A, et al. Cerebrovascular accidents indicative of COVID-19 infection: about 4 observations in Guinea. Pan Afr Med J 2020; 35: 65. DOI: 10.11604/pamj.supp.2020.35.2.23751. 